
https://doi.org/10.1007/s41969-020-00121-w Online publiziert: 30 . Oktober 2020 (c) Der/die Autor(en) 2020
J. Klin. Endokrinol. Stoffw. $2020 \cdot 13: 151-155$

\author{
Alexander Micko' - Stefan Wolfsberger ${ }^{1} \cdot$ Greisa Vila $^{2} \cdot$ Romana Höftberger ${ }^{3}$. \\ Anton Luger ${ }^{2} \cdot$ Engelbert Knosp' \\ ${ }^{1}$ Universitätsklinik für Neurochirurgie, Medizinische Universität Wien, Wien, Österreich \\ ${ }^{2}$ Universitätsklinik für Innere Medizin III, Abteilung für Endokrinologie und Stoffwechsel, Medizinische \\ Universität Wien, Wien, Österreich \\ ${ }^{3}$ Universitätsklinik für Neurologie, Abteilung für Neuropathologie und Neurochemie, Medizinische \\ Universität Wien, Wien, Österreich
}

\section{Aktueller Stand der Hypophysenchirurgie}

\section{Überblick}

Hypophysenadenome machen $15 \%$ aller intrakraniellen Tumoren aus und treten mit einer Inzidenz von 2,7/100.000 und Jahr auf [1]. Mit $65 \%$ sind hormonaktive Adenome häufiger als hormoninaktive $(35 \%)$. Hormonaktive Adenome setzen sich zusammen aus: PRL-produzierenden Adenomen (25-41\%), GH-ZellAdenomen (10-15\%), ACTH-Zell-Adenomen (15\%) und TSH-Zell-Adenomen $(2 \%)[1,2]$.

Die chirurgische Entfernung ist trotz Verbesserungen im Bereich der medikamentösen Therapie als auch der Strahlentherapie weiterhin die Therapie der ersten Wahl bei allen Hypophysenadenomen, ausgenommen den PRL-produzierenden Adenomen, die standardmäßig medikamentös mit Dopaminagonisten behandelt werden.

Eine chirurgische Behandlung kann bei bestimmten Prolaktinomen in Erwägung gezogen werden:

1. bei zystischen bzw. eingebluteten Prolaktinomen, die einen Masseneffekt oder eine Apoplexie mit Sehverlust verursachen,

2. bei ausgeprägten Nebenwirkungen bzw. ungenügendem Ansprechen auf die Dopaminagonistentherapie [2-7],

3. bei nichtinvasiven Mikroprolaktinomen auf ausdrücklichen Patientenwunsch als Alternative zur meist lebenslangen medikamentösen Behandlung [8].

Technologische Fortschritte haben die chirurgische Behandlung von Hypophy- senadenomen in den letzten Jahren sicherer und effektiver gemacht:

\section{Transnasale Endoskopie (o Abb. 1)}

Der chirurgische Zugang zur Resektion von Hypophysenadenomen - nämlich, ob auf transkraniellem oder transsphenoidalem Weg - war vor Jahrzehnten noch ein Diskussionsthema. Mittlerweile ist jedoch der transnasale, transsphenoidale Zugang der weltweit am meisten angewandte und akzeptierte Zugangsweg [9]. Die Diskussion dreht sich nun um die Frage, ob der traditionell mikroskopischen oder neu eingeführten endoskopischen Visualisierungstechnik der Vorzug gegeben werden soll. Während der endoskopische Ansatz eine direkte Sicht, ein breiteres Sichtfeld und den Blick auf seitliche Strukturen mittels Winkeloptiken liefert, besteht der Nachteil darin, dass die Tiefenwahrnehmung durch ein zweidimensionales Bild erschwert wird $[10,11]$.

Trotz dieser offensichtlichen Vorteile der Endoskopie konnte eine rezente Metaanalyse keine signifikante Verbesserung in Bezug auf Komplikationen und Heilungsrate finden [12].

Jedoch kann v.a. bei ausgedehnten und komplex konfigurierten Hypophysenadenomen durch das Endoskop und damit möglich gewordene „Extended“Zugänge ein höheres Maß an Tumorresektion erzielt werden.

\section{Invasivität und die daraus resultierenden Probleme (- Abb. 2)}

Die Invasivität von Hypophysenadenomen ist unabhängig von der Operationstechnik der limitierende Faktor der chirurgischen Heilung. Invasivität ist defi-

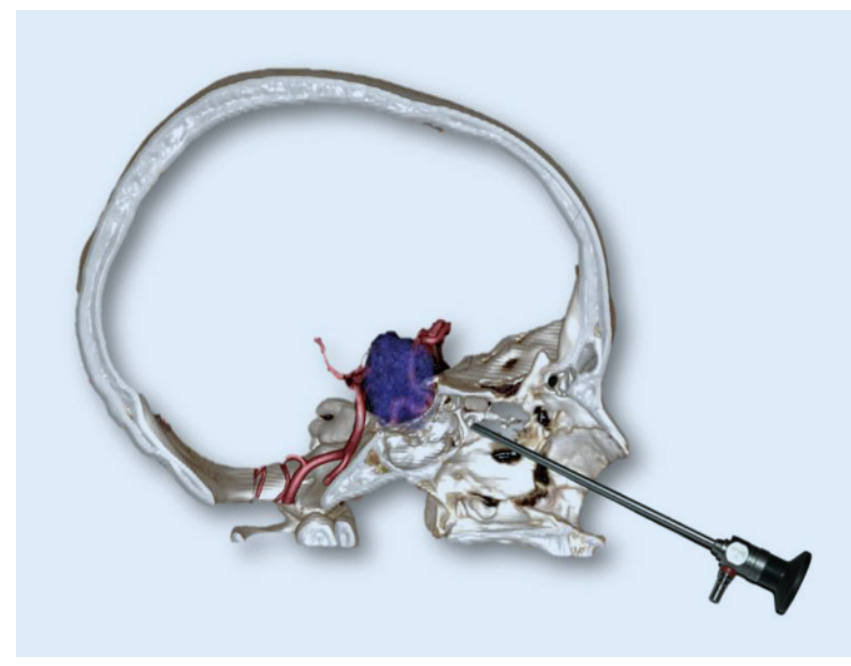

Abb. $1<$ Schematische Darstellung der endoskopischen Resektion eines Hypophysenmakroadenoms. (Copyright: DDr. A. Micko) 

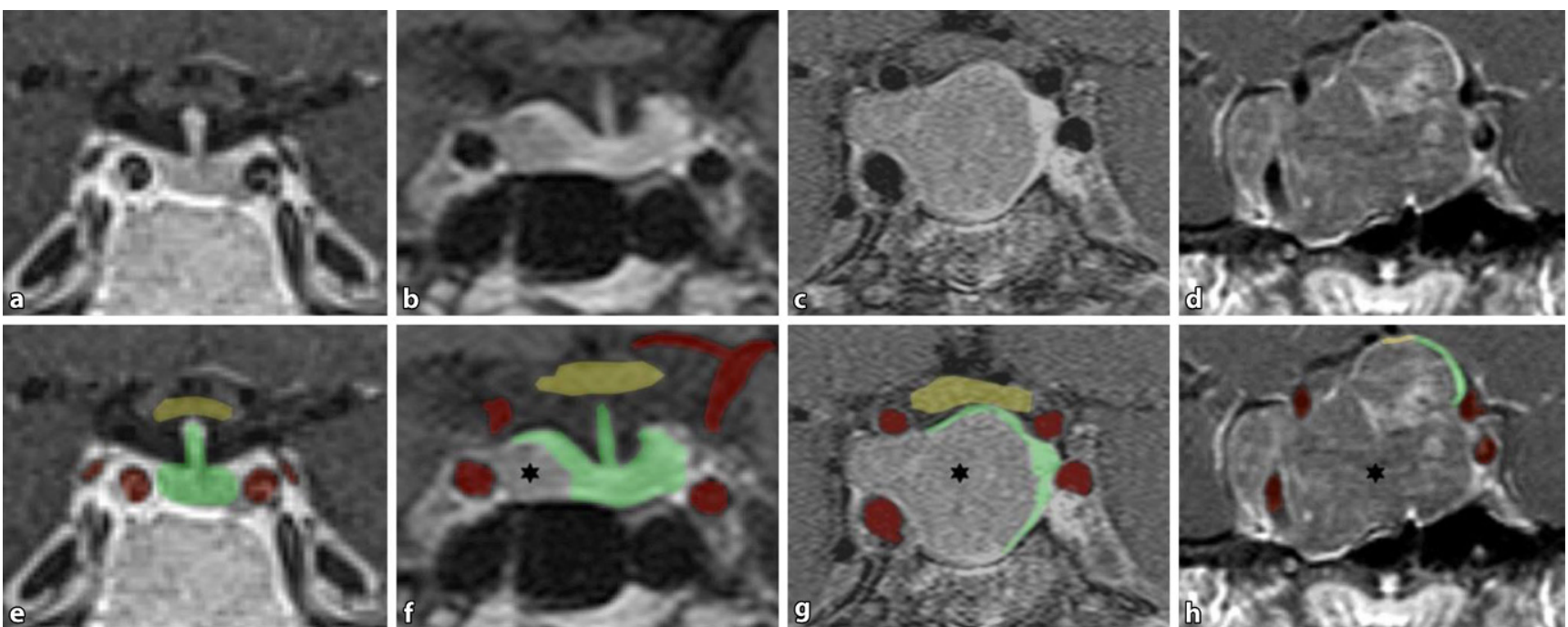

Abb. $2 \Delta$ a Normale Hypophyse ohne Anzeichen eines Hypophysenadenoms, b Mikroadenom ( $<1 \mathrm{~cm}$ Durchmesser), rechts endosellar, c Makroadenom (>1 cm) mit parasellärer Ausdehnung, jedoch ohne Anzeichen einer Invasivität, d Makroadenom mit Anzeichen einer parasellären Invasivität, e- $\mathbf{h}$ grafische Visualisierung der anatomischen Struktur. Asteriskus Hypophysenadenom, grün normale Hypophyse, rot A. carotis interna, gelb Chiasma opticum. (Copyright: DDr. A. Micko)

niert als infiltratives Wachstum in Dura, Knochenstrukturen und paraselläre Bereiche wie den seitlich angrenzenden Sinus cavernosus.

Paraselläres Wachstum tritt bei ca. $6-10 \%$ aller Hypophysenadenome auf, in chirurgischen Serien wurden jedoch Anzeichen einer Invasivität in bis zu $40 \%$ der Fälle beschrieben [13-17]. Die genaue Kenntnis der parasellären Anatomie des Sinus cavernosus ist für Operationen in diesem Bereich von entscheidender Bedeutung. Basierend auf den Pionierarbeiten von Parkinson und Dolenc wurde die Operation von Tumoren, die in den Raum auftreten, mit geringen Komplikationsraten möglich [18-20].

Die mediale Wand des Sinus cavernosus ist dabei eine kritische Struktur; sie ist eine dünne Membran, die an den lateralen Teil der Hypophyse angrenzt. Nur die A. hypophysialis inferior und die Hypophysenvenen verlaufen durch diese Wand. Alle sonstigen Öffnungen in der medialen Wand des Sinus cavernosus, die während der Operation beobachtet werden, sind durch ein invasives Tumorwachstum verursacht. Invasive Hypophysenadenome neigen dazu, sich durch die mediale Wand im Sinus cavernosus auszubreiten.

Abhängig von verdrängendem oder invasivem Adenomwachstum wird die im
Sinus cavernosus verlaufende A. carotis interna durch den Tumor entweder verdrängt oder invasiv umschlossen.

Die Klassifikation nach Knosp ist wichtig, weil sie die paraselläre $\mathrm{Tu}$ morausdehnung auf den präoperativen MRT-Bildern in Bezug auf die Lage der A. carotis interna beurteilt: Je höher der Knosp-Grad ist, desto schwieriger ist eine vollständige chirurgische Entfernung, und desto wahrscheinlicher ist eine postoperative medikamentöse oder radiochirurgische Therapie [16, 21].

Daher ist v. a. bei invasiven Hypophysenadenomen ein interdisziplinäres Vorgehen, welches nur in großen Zentren möglich ist, zur Behandlung dieser Tumoren nötig.

\section{Intraoperative Bildgebung}

\section{Neuronavigation}

Präzision ist entscheidend für den Erfolg eines neurochirurgischen Eingriffs. Daher wurde in den letzten 2 Jahrzehnten in vielen Teilgebieten der Neurochirurgie die Neuronavigation implementiert und etabliert [22-27]. In der transnasalen transsphenoidalen Hypophysenchirurgie erhalten Neurochirurgen durch die Neuronavigation die Möglichkeit, intraund paraselläre Strukturen intraoperativ zu verifizieren [28]. Die Registrierung ist dabei der Schlüsselschritt in der navigationsgesteuerten Chirurgie und wird definiert als die Bestimmung einer geometrischen Transformation, die die Bildgebung des Patienten mit dem physischen Patienten in Beziehung setzt [29]. So kann die räumliche Position eines verfolgten Instruments relativ zum Patienten (über einen am Kopf oder an der Kopffixierungsklemme angebrachten Tacker) während des Eingriffs bestimmt werden.

Unter Zuhilfenahme der Neuronavigation konnte gezeigt werden, dass durch die verbesserte Orientierung die Rate an Komplikationen bei komplexen Fällen, aber v. a. bei Rezidiveingriffen gesenkt werden kann [30].

\section{Intraoperatives Magnetresonanz- tomogramm}

Ziel der chirurgischen Resektion von Hypophysenadenomen ist es, das Ausmaß der Tumorresektion zu maximieren, während die Funktion der Hypophyse erhalten bleibt. Die Lage eines möglichen Adenomrests kann aber vom Chirurgen während des Eingriffs nicht immer sicher festgestellt werden.

Das intraoperative Magnetresonanztomogramm (MRT) ermöglicht dem Chirurgen eine hochauflösende Darstellung des Resektionsausmaßes bereits während des Eingriffs. Dies ist v. a. bei 
Hier steht eine Anzeige.

\section{曾 Springer}


großen und komplex konfigurierten Tumoren von Vorteil, um Adenomreste zu lokalisieren. Zusätzlich können durch die intraoperative Bildgebung die Neuronavigation aktualisiert werden und Komplikationen wie z.B. Tumoreinblutungen bereits während des Eingriffs antizipiert werden [31-33].

Der Nachteil dieser Technologie sind jedoch die hohen Anschaffungs- und Erhaltungskosten.

\section{Biologische Marker}

Ein wichtiger, die Remissionsrate von Hypophysenadenomen beeinflussender Faktor ist die Expression von Proliferationsmarkern. Das Ki-67(MIB-1)-Protein ist ein Proliferationsmarker, der in einer Reihe von Tumorentitäten (z.B. Brustund Prostatakrebs, neuroendokrine Tumoren und Hirntumoren) angewendet wird, um den Grad der Zellproliferation während aller aktiven Phasen des Zellzyklus zu erfassen [34].

In der aktuellen WHO-Klassifikation von 2017 wurde dieser Proliferationsmarker zwar als Kriterium einer erhöhten Tumoraktivität gewertet, jedoch wurden klare Grenzwerte, um aggressive Hypophysenadenome zu klassifizieren, nicht festgesetzt. Aufgrund mangelnder Reproduzierbarkeit in mehreren Studien, die zu dieser Entscheidung der WHO geführt hatten, wurde durch Trouillas et al. eine multizentrische Fall-KontrollStudie mit klaren Grenzwerten durchgeführt. In ihrer Analyse zeigten Hypophysenadenome, die ein invasives Wachstum sowie die Expression von Proliferationsmarkern zeigen, ein hohes Risiko für ein Wiederauftreten oder Fortschreiten eines Resttumors [14].

Darüber hinaus konnte bei Tumoren, die eine Kombination mehrerer Transkriptionsfaktoren aufweisen, ein erhöhtes Risiko für ein aggressives Verhalten nachgewiesen werden [35].

\section{Gamma-Knife}

Radiochirurgie und externe Strahlentherapie werden als zusätzliche Behandlungen von Hypophysenadenomen eingesetzt, falls folgende Kriterien zutreffen: unvollständige chirurgische Resektion,

J. Klin. Endokrinol. Stoffw. 2020 · 13:151-155 https://doi.org/10.1007/s41969-020-00121-w (c) Der/die Autor(en) 2020

A. Micko · S. Wolfsberger · G. Vila · R. Höftberger · A. Luger · E. Knosp

Aktueller Stand der Hypophysenchirurgie

\section{Zusammenfassung}

Eine gut funktionierende Kooperation zwischen Neurochirurgie und Endokrinologie sowie die enge Zusammenarbeit mit Neuropathologie, Neuroradiologie und Neuroophthalmologie sind die Voraussetzungen für eine erfolgreiche Behandlung von Hypophysenadenomen. Hypophysenadenome können heute durch den technologischen Fortschritt immer sicherer und komplikationsärmer operiert werden. Dazu gehört neben einer High-Tech-Ausrüstung auch eine spezielle histologische bzw. molekulare
Aufarbeitung des Tumorgewebes, um eine optimale multimodale Therapie anbieten zu können. Um ausgedehnte, invasive und aggressiv wachsende Hypophysenadenome, die ein interdisziplinäres Vorgehen benötigen, behandeln zu können, ist es heutzutage notwendig, dies in spezialisierten Zentren durchzuführen.

Schlüsselwörter

Hypophysenadenom - Resektion .

Endoskopisch · Invasivität · Zentrum

\section{Current state of pituitary gland surgery}

\section{Abstract}

A well-functioning cooperation between neurosurgery and endocrinology and a close collaboration with neuropathology, neuroradiology and neuro-ophthalmology are prerequisites for successful treatment of pituitary adenomas. Due to the technological progress, pituitary adenomas can nowadays be more safely surgically treated and with a continuous reduction in the number of complications. In addition to high-tech equipment, this includes a special histological and molecular processing of the tumor tissue, in order to be able to provide an optimal multimodal treatment. In order to be able to treat extensive, invasive and aggressively growing pituitary adenomas, which necessitate an interdisciplinary approach, it is nowadays necessary that this is carried out in specialized centers.

\section{Keywords}

Pituitary adenoma - Resection - Endoscopic . Invasiveness - Center
Wiederauftreten des Tumors oder Versagen der medikamentösen Behandlung.

Im Falle einer externen Strahlentherapie von Hypophysenadenomen besteht das Behandlungsschema aus einer Gesamtstrahlungsdosis von $45-54 \mathrm{~Gy}$, die in 25- bis 30-täglichen Fraktionen abgegeben wird; diese wird hauptsächlich eingesetzt, wenn größere Tumoranteile sich am Sehnerv befinden, die chirurgisch nicht mehr reseziert werden können [36].

Im Gegensatz dazu hat die GammaKnife-Radiochirurgie (GKN) den Vorteil einer Einzelsitzungstherapie mit hochkonzentrierten Dosen, die an das Ziel abgegeben werden. Die GKN verwendet mehrere Isozentren, um aufgrund unterschiedlicher anatomischer Bedingungen einen fokussierten Dosisplan zu erstellen. Ein steiler Dosisabfall kann dadurch das umgebende Gewebe schonen. Aufgrund der fehlenden medikamentösen Alternative bleibt sie die Zweitlinienbehandlung für nichtfunktionell aktive Strahlentherapie. Eine Kontrolle des Resttumorvolumens kann dabei in $50-80 \%$ der Fälle erreicht werden [37].

Als Third-Line-Therapie ist die Radiochirurgie bei GH-produzierenden Adenomen im Falle einer parasellären Invasivität besonders wichtig. In der Literatur gibt es eine große Variabilität der endokrinen Remissionsraten (0-100\%) sowie des Zeitintervalls zwischen der radiochirurgischen Behandlung und der endokrinen Remission (3 Monate bis 8 Jahre) [36, 38, 39].

Die häufigste Nebenwirkung der Radiochirurgie ist jedoch die hypophysäre Insuffizienz, die in bis zu $70 \%$ der Fälle auftritt und dosisabhängig ist, gefolgt von einer in ca. $4 \%$ neu aufgetretenen Seheinschränkung [40]. 


\section{Korrespondenzadresse}

\section{Assoc. Prof. Priv.-Doz. Dr.}

\section{Stefan Wolfsberger}

Universitätsklinik für Neurochirurgie,

Medizinische Universität Wien

Währinger Gürtel 18-20, 1090 Wien, Österreich

stefan.wolfsberger@meduniwien.ac.at

\section{Prof. Dr. Engelbert Knosp}

Universitätsklinik für Neurochirurgie,

Medizinische Universität Wien

Währinger Gürtel 18-20, 1090 Wien, Österreich engelbert.knosp@meduniwien.ac.at

Funding. Open access funding provided by Medical University of Vienna.

\section{Einhaltung ethischer Richtlinien}

Interessenkonflikt. A. Micko, S. Wolfsberger, G. Vila, R. Höftberger, A. Luger und E. Knosp geben an, dass kein Interessenkonflikt besteht.

Für diesen Beitrag wurden von den Autoren keine Studien an Menschen oder Tieren durchgeführt. Für die aufgeführten Studien gelten die jeweils dort angegebenen ethischen Richtlinien.

Open Access Dieser Artikel wird unter der Creative Commons Namensnennung 4.0 International Lizenz veröffentlicht, welche die Nutzung, Vervielfältigung Bearbeitung, Verbreitung und Wiedergabe in jeglichem Medium und Format erlaubt, sofern Sie den/die ursprünglichen Autor(en) und die Quelle ordnungsgemäß nennen, einen Link zur Creative Commons Lizenz beifügen und angeben, ob Änderungen vorgenommen wurden.

Die in diesem Artikel enthaltenen Bilder und sonstiges Drittmaterial unterliegen ebenfalls der genannten Creative Commons Lizenz, sofern sich aus der Abbildungslegende nichts anderes ergibt. Sofern das betreffende Material nicht unter der genannten Creative Commons Lizenz steht und die betreffende Handlung nicht nach gesetzlichen Vorschriften erlaubt ist, ist für die oben aufgeführten Weiterverwendungen des Materials die Einwilligung des jeweiligen Rechteinhabers einzuholen.

Weitere Details zur Lizenz entnehmen Sie bitte de Lizenzinformation auf http://creativecommons.org/ licenses/by/4.0/deed.de.

\section{Literatur}

1. MelmedS (2011) Pathogenesis of pituitary tumors Nat Rev Endocrinol 7(5):257-266

2. Colao A, Savastano S (2011) Medical treatment of prolactinomas. Nat Rev Endocrinol 7(5):267-278

3. Couldwell WT, Rovit RL, Weiss MH (2003) Role of surgery in the treatment of microprolactinomas. Neurosurg Clin N Am 14(1):89-92

4. Liu JK, Couldwell WT (2004) Contemporary management of prolactinomas. Neurosurg Focus 16(4):E2
5. OhMC, KunwarS, Blevins L, Aghi MK (2012)Medical versus surgical management of prolactinomas. Neurosurg Clin N Am 23(4):669-678

6. Olafsdottir A, Schlechte J (2006) Management of resistant prolactinomas. Nat Clin Pract Endocrinol Metab 2(10):552-561

7. Zygourakis CC, Imber BS, Chen Ret al (2017) Costeffectiveness analysis of surgical versus medical treatment of prolactinomas. J Neurol Surg B Skull Base 78(2):125-131

8. Dekkers OM, Lagro J, Burman P et al (2010) Recurrence of hyperprolactinemia after withdrawal of dopamine agonists: systematic review and metaanalysis. JClin Endocrinol Metab 95(1):43-51

9. Liu JK, Das K, Weiss MH, Laws ER Jr., Couldwell WT (2001) The history and evolution of transsphenoidal surgery. J Neurosurg 95(6):1083-1096

10. Cappabianca P, Cavallo LM, de Divitiis E (2004) Endoscopic endonasal transsphenoidal surgery. Neurosurgery55(4):933-940 (discussion 940-931)

11. Jho HD (2001) Endoscopic transsphenoidal surgery. J Neurooncol 54(2):187-195

12. Ammirati M, Wei L et al (2013) Short-term outcome of endoscopic versus microscopic pituitary adenoma surgery: a systematic review and meta-analysis. J Neurol Neurosurg Psychiatry 84(8):843-849

13. Fahlbusch R, Buchfelder M (1988) Transsphenoida surgery of parasellar pituitary adenomas. Acta Neurochir 92(1-4):93-99

14. Trouillas J, Roy P, Sturm N et al (2013) A new prognostic clinicopathological classification of pituitary adenomas: a multicentric case-control study of 410 patients with 8 years post-operative follow-up. Acta Neuropathol 126(1):123-135

15. Vieira JO Jr., Cukiert A, Liberman B (2006) Evaluation of magnetic resonance imaging criteria for cavernous sinus invasion in patients with pituitary adenomas: logistic regression analysis and correlation with surgical findings. Surg Neurol 65(2):130-135 (discussion 135)

16. Knosp E, Steiner E, Kitz K, Matula C (1993) Pituitary adenomas with invasion of the cavernous sinus space: a magneticresonance imaging classification compared with surgical findings. Neurosurgery 33(4):610-617 (discussion 617-618)

17. Kitano M, Taneda M, Shimono T, Nakao Y (2008) Extended transsphenoidal approach for surgical management of pituitary adenomas invading the cavernous sinus. J Neurosurg 108(1):26-36

18. Parkinson D (1990) Surgical anatomy of the lateral sellar compartment (cavernous sinus). Clin Neurosurg 36:219-239

19. Dolenc V (1989) Anatomy of the cavernous sinus. In: Anatomy and surgery of the cavernous sinus. Springer, Vienna, S3-137

20. Frank G, Pasquini E (2006) Endoscopic endonasal cavernous sinus surgery, with special reference to pituitaryadenomas. Front Horm Res 34:64-82

21. Micko AS et al (2015) Invasion of the cavernous sinus space in pituitary adenomas: endoscopic verification and its correlation with an MRI-based classification. J Neurosurg 122(4):803-811

22. Brinker T, Arango G et al (1998) An experimental approach to image guided skull base surgery employing a microscope-based neuronavigation system. Acta Neurochir 140(9):883-889

23. Ende G, Treuer H, Boesecke R (1992) Optimization and evaluation of landmark-based image correlation. Phys Med Biol 37(1):261-271

24. Glossop ND (2009) Advantages of optical compared with electromagnetic tracking. J Bone Joint Surg Am 91 (Suppl 1):23-28

25. Hayhurst C, Byrne P, Eldridge PR, Mallucci CL (2009) Application of electromagnetic technology to neuronavigation: a revolution in image-guided neurosurgery. J Neurosurg 111(6):1179-1184

26. Shamir RR, Joskowicz L, Spektor S, Shoshan Y (2009) Localization and registration accuracy in image guided neurosurgery: a clinical study. Int Comput Assist Radiol Surg 4(1):45-52

27. Wolfsberger S, Rossler K, Regatschnig R, Ungersbock K (2002) Anatomical landmarks for image registration in frameless stereotactic neuronavigation. Neurosurg Rev 25(1-2):68-72

28. McGrath BM, Maloney WJ, Wolfsberger S et a (2010) Carotid artery visualization during anterior skull base surgery: a novel protocol for neuronavigation. Pituitary 13(3):215-222

29. Fitzpatrick JM (2010) The role of registration in accurate surgical guidance. Proc Inst Mech Eng $\mathrm{H}$ 224(5):607-622

30. Micko A, Hosmann A, Wurzer A et al (2019) An advanced protocol for intraoperative visualization of sinunasal structures: experiences from pituitary surgery. J Neurosurg. https://doi.org/10.3171/ 2019.3.JNS1985

31. Buchfelder M, Schlaffer SM (2012) Intraoperative magnetic resonance imaging during surgery fo pituitary adenomas: pros and cons. Endocrine 42(3):483-495

32. Schwartz TH, Stieg PE, Anand VK (2006) Endoscopic transsphenoidal pituitary surgery with intraoperative magnetic resonance imaging. Neurosurgery 58(1 Suppl):ONS44-ONS51

33. Theodosopoulos PV, Leach J, Kerr RG et al (2010) Maximizing the extent of tumor resection during transsphenoidal surgery for pituitary macroadenomas: can endoscopy replace intraoperative magnetic resonance imaging? J Neurosurg 112(4):736-743

34. Cattoretti G, Becker MH, Key G et al (1992 Monoclonal antibodies against recombinan parts of the Ki-67 antigen (MIB 1 and MIB 3) detect proliferating cells in microwaveprocessed formalin-fixed paraffin sections.J Pathol 168(4):357-363

35. Micko A, Rotzer T, Hoftberger R et al (2020) Expression of additional transcription factors is of prognostic value for aggressive behavior of pituitary adenomas. J Neurosurg. https://doi.org/ 10.3171/2020.2.JNS2018

36. Ding D, Starke RM, Sheehan JP (2014) Treatment paradigms for pituitary adenomas: defining the roles of radiosurgery and radiation therapy. JNeurooncol 117(3):445-457

37. Hoybye C, Rahn T (2009) Adjuvant Gamma Knife radiosurgery in non-functioning pituitary adenomas; low risk of long-term complications in selected patients. Pituitary 12(3):211-216

38. Sheehan JM, Vance ML, Sheehan JP, Ellegala DB, Laws ER Jr. (2000) Radiosurgery for Cushing's disease after failed transsphenoidal surgery. JNeurosurg 93(5):738-742

39. Tinnel BA, Henderson MA, Witt TC et al (2008) Endocrine response after gamma knife-based stereotactic radiosurgery for secretory pituitary adenoma. Stereotact Funct Neurosurg 86(5):292-296

40. Sheehan JP, Starke RM, Mathieu D et al (2013) Gamma Knife radiosurgery for the management of nonfunctioning pituitary adenomas: a multicenter study. J Neurosurg 119(2):446-456

Hinweis des Verlags. Der Verlag bleibt in Hinblick auf geografische Zuordnungen und Gebietsbezeichnungen in veröffentlichten Karten und Institutsadressen neutral. 